\title{
Ontogeny of glucocorticoid receptor and 11ß-hydroxysteroid dehydrogenase type-1 gene expression identifies potential critical periods of glucocorticoid susceptibility during development
}

\author{
H J L Speirs, J R Seckl and R W Brown \\ Molecular Medicine Centre, Western General Hospital, Edinburgh EH4 2XU, UK \\ (Requests for offprints should be addressed to R Brown; Email: Roger.Brown@ed.ac.uk)
}

\begin{abstract}
Glucocorticoids play important roles in organ development and 'fetal programming'. Fetal exposure to excess glucocorticoids reduces birth weight and causes later hypertension. To investigate these processes further we have determined the detailed ontogeny in the mouse of the glucocorticoid receptor (GR) and 11ß-hydroxysteroid dehydrogenase type-1 (11 $\beta$-HSD1), which amplifies glucocorticoid levels locally; the ontogeny was determined using in situ hybridisation from embryonic day 9.5 (E9.5, term $=$ E19) until after birth.

At E9.5 fetal GR mRNA levels are very low, except in fetal placenta. GR gene expression rises during gestation with striking tissue-specific differences in timing and extent. Before E13.5, an increase is clear in gastrointestinal (GI) and upper respiratory tracts, discrete central nervous system (CNS) regions, precartilage and especially in the liver (E10-5-E12). Later, further increases occur in lung, GI and upper respiratory tracts, muscle, pituitary and
\end{abstract}

thymus. In a few tissues such increases are temporary, e.g. ureteric ducts (E13.5-E16.5) and pancreas (E14.5-E16.5, expression later falling sharply).

Fetal $11 \beta-H S D 1$ mRNA expression is first clearly observed at E14.5-E15, initially in the fetal placenta then in the umbilical cord. Later, $11 \beta-H S D 1$ expression is seen as follows: (i) from E15 in lung and liver, rising strongly; (ii) thymus, from E15 (lower level); (iii) at low levels in a few brain regions, including the hippocampus (E16.5+); and (iv) in muscle group fascial planes and tendon insertions.

This is the first detailed study of the ontogeny of these two genes and, in combination with previous work on the ontogeny of $11 \beta-H S D 2$ and the mineralocorticoid receptor, suggests potential critical periods of glucocorticoid sensitivity during development for several organ systems. Journal of Endocrinology (2004) 181, 105-116

\section{Introduction}

Glucocorticoid hormones play many critical roles in both normal physiology and the stress response. Prenatally, glucocorticoids are important, not only in the structural development of organs but also in the functional maturation of many key tissues, notably in preparation for birth. Indeed, glucocorticoids are used clinically to accelerate the maturation of the premature fetal lung. Mice homozygous for targeted disruption of the glucocorticoid receptor (GR) gene die at birth because of a lack of normal lung maturation (Cole et al. 1995). However, there can be potentially detrimental longer-term consequences of glucocorticoid overexposure in utero. In particular, exposure to synthetic glucocorticoids reduces birth weight (Edwards et al. 1993, Seckl et al. 2000). Multiple studies associate low birth weight with a substantially increased risk of cardiovascular and metabolic disorders in adult life. Indeed, excess prenatal glucocorticoid exposure in rats models these associations, reducing birth weight and

permanently 'programming' abnormalities in the offspring; these abnormalities manifest in adulthood as hypertension, impaired glucose tolerance with hyperinsulinaemia and abnormalities of the hypothalamopituitary-adrenal axis and behaviour (Seckl et al. 2000). Clearly tight physiological control of fetal (and placental) glucocorticoid exposure is crucial to prevent such adverse consequences.

Physiological glucocorticoids act via nuclear receptors of two types: a high affinity type-1 receptor (MR, also binding aldosterone with equal affinity) and a lower affinity glucocorticoid or type-2 receptor (GR). Variation in tissue sensitivity to glucocorticoids is determined not only by the levels of these two receptors but also by local glucocorticoid activation/inactivation by two key intracellular enzymes (11 $\beta$-hydroxysteroid dehydrogenase

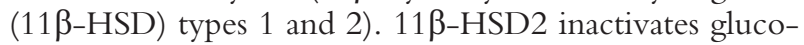
corticoids, so greatly limiting glucocorticoid access to co-localised GR and MR. 11 $\beta-H S D 2$ has an adult tissue distribution that is largely, but not exclusively, restricted to 
MR-expressing aldosterone target tissues (e.g. distal nephron) (Brown et al. 1996a). When 11ß-HSD2 is abundant this precludes glucocorticoid access to MR but allows the 100- to 1000-fold lower circulating levels of aldosterone to pass unmetabolised and bind MR selectively. In humans, deficiencies of $11 \beta-H S D 2$ in kidney allows cortisol access to MR causing apparent mineralocorticoid excess and hypertension. In contrast 11 $\beta-$ HSD1 acts as a reductase locally regenerating glucocorticoids. $11 \beta-H S D 1$ is frequently co-localised with GR in adult tissues, both having a broad tissue distribution in adults (Whorwood et al. 1992). Thus, GR and 11ß-HSD1 are seen as playing a major role in modulating the level of glucocorticoid-dependent responses in a wide range of adult tissues.

To elucidate the ontogeny of corticosteroid responsiveness we have previously mapped gene expression of MR and $11 \beta-H S D 2$ during mouse development (Brown et al. 1996c). This revealed high placental $11 \beta-H S D 2$ expression, while in the fetus a pattern beginning to resemble adult MR and $11 \beta-H S D 2$ expression appeared only in the late-gestation/neonatal period. Earlier in gestation the expression of these two genes was completely different as MR gene expression was very limited/absent, while $11 \beta-H S D 2$ was expressed very widely in fetal tissues at high level. Such high placental and fetal 11ß-HSD2 activity affords protection to the developing fetus from the much higher maternal glucocorticoid levels (Edwards et al. 1993, Seckl \& Brown 1994). Protection from excess glucocorticoid exposure during development is clearly important. Thus, individuals homozygous for deleterious mutations of the $11 \beta-\mathrm{HSD} 2$ gene (Mune et al. 1995), or the use of $11 \beta-H S D 2$ inhibitors in pregnant rats, cause dramatic reductions in birth weight (Seckl et al. 2000). The adult rats have hypertension, hyperglycaemia and central nervous system (CNS) changes similar to those seen with gestational exposure to excess synthetic glucocorticoids (poor substrates for $11 \beta-\mathrm{HSD} 2$ ).

To further clarify the ontogeny of glucocorticoid responsiveness we present a complementary in situ hybridisation study in the mouse of the ontogeny of GR and the glucocorticoid-activating enzyme $11 \beta-H S D 1$ from embryonic day $9 \cdot 5(E 9 \cdot 5$, term $=$ E19) until after birth. This study reveals striking changes in the expression of both genes with 11ß-HSD1 only appearing in latergestation fetal tissues; the ontogeny of GR gene expression is shown to be very dynamic, with a number of striking increases that are tissue-specific and arise within limited developmental time windows.

\section{Materials and Methods}

\section{Animals}

C57BL/6 mice were grouped for mating and were checked the following morning. When mating had

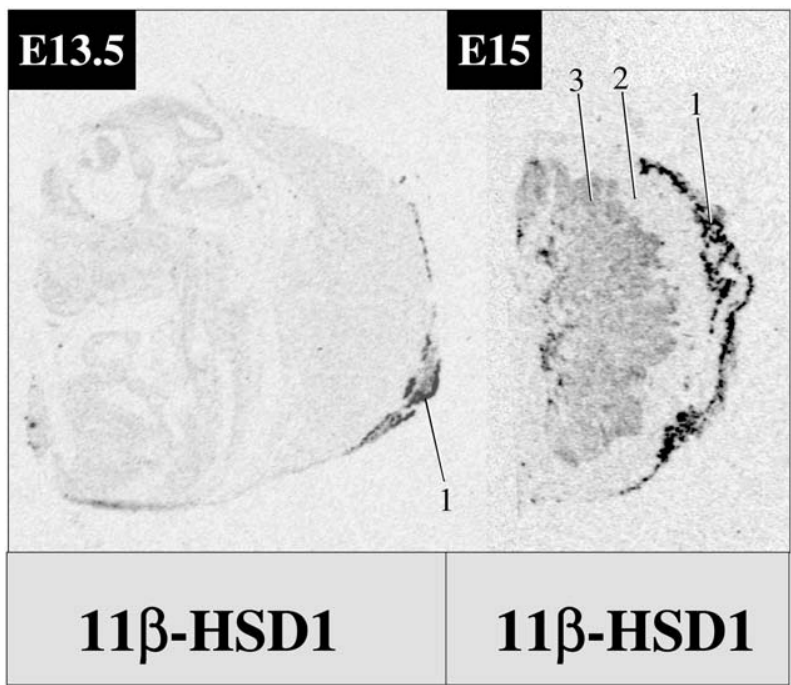

Figure 1 Initial expression of $11 \beta$-HSD 1 during mouse development. 1 , uterine wall; 2 , decidua; 3 , fetal zone of placenta.

occurred females were designated as potentially pregnant at gestation E0.5. This allowed embryos at appropriate gestational ages (from E9.5) and pups (0.5 days postnatal, $\mathrm{P} 0 \cdot 5)$ to be obtained; these were frozen immediately $\left(-20{ }^{\circ} \mathrm{C}\right.$ isopentane bath) after death and were stored at $-80{ }^{\circ} \mathrm{C}$. Litters from 25 dams were collected having equal gender ratio and 5-11 pups per litter. Crown-rump length and morphological correlation with embryonic (Theiler) staging milestones allowed verification of actual embryonic stage. All work was carried out under regulatory animal licence to ensure humane standards at all times.

\section{$11 \beta$-HSD1 and GR probes}

Separate cDNA constructs incorporating mouse $11 \beta$ HSD1 or GR cDNA inserts flanked by T3 and T7 polymerase binding sites were generated by PCR (insert identities confirmed by sequencing) allowing production

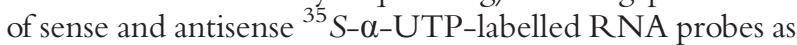
described previously for other genes (Brown et al. 1996a, Macdonald et al. 2000). The GR construct (cGR) allowed generation of $337 \mathrm{bp}$ mouse GR probes corresponding to the $3^{\prime}$ untranslated region of mouse GR (running $3^{\prime}$ from bp 2501 in mouse GR sequence X04435 (Danielsen et al. 1986) and corresponding to bp 55-391 from BE377537; this showed $90 \%$ identity to the rat GR $\alpha$ sequence (M14053 bp 2590-2926)). For 11 $\beta$-HSD1 two constructs were generated: (i) c11 $\beta 1$, producing $422 \mathrm{bp}$ probes (to $\mathrm{bp}$ 32-453 from AI097870) to the $5^{\prime}$ untranslated region of exon 1A through to just below the exon 3-4 splice junction; and (ii) c11 $\beta 1 \mathrm{~A}$, producing $178 \mathrm{bp}$ probes specific to exon 1A (bp 32-209 from the same sequence). 

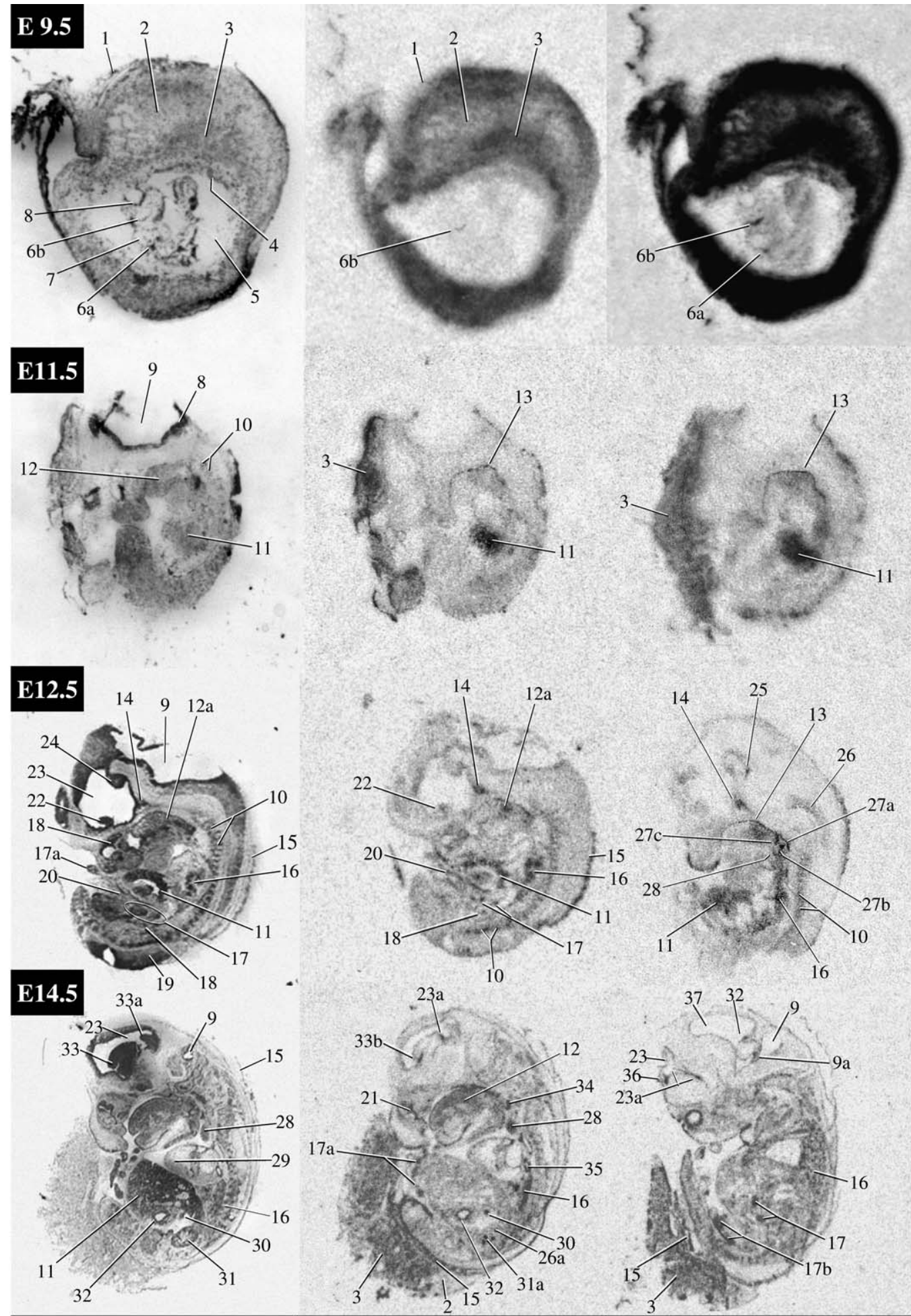

Stained

GR

GR

Figure 2(a). 


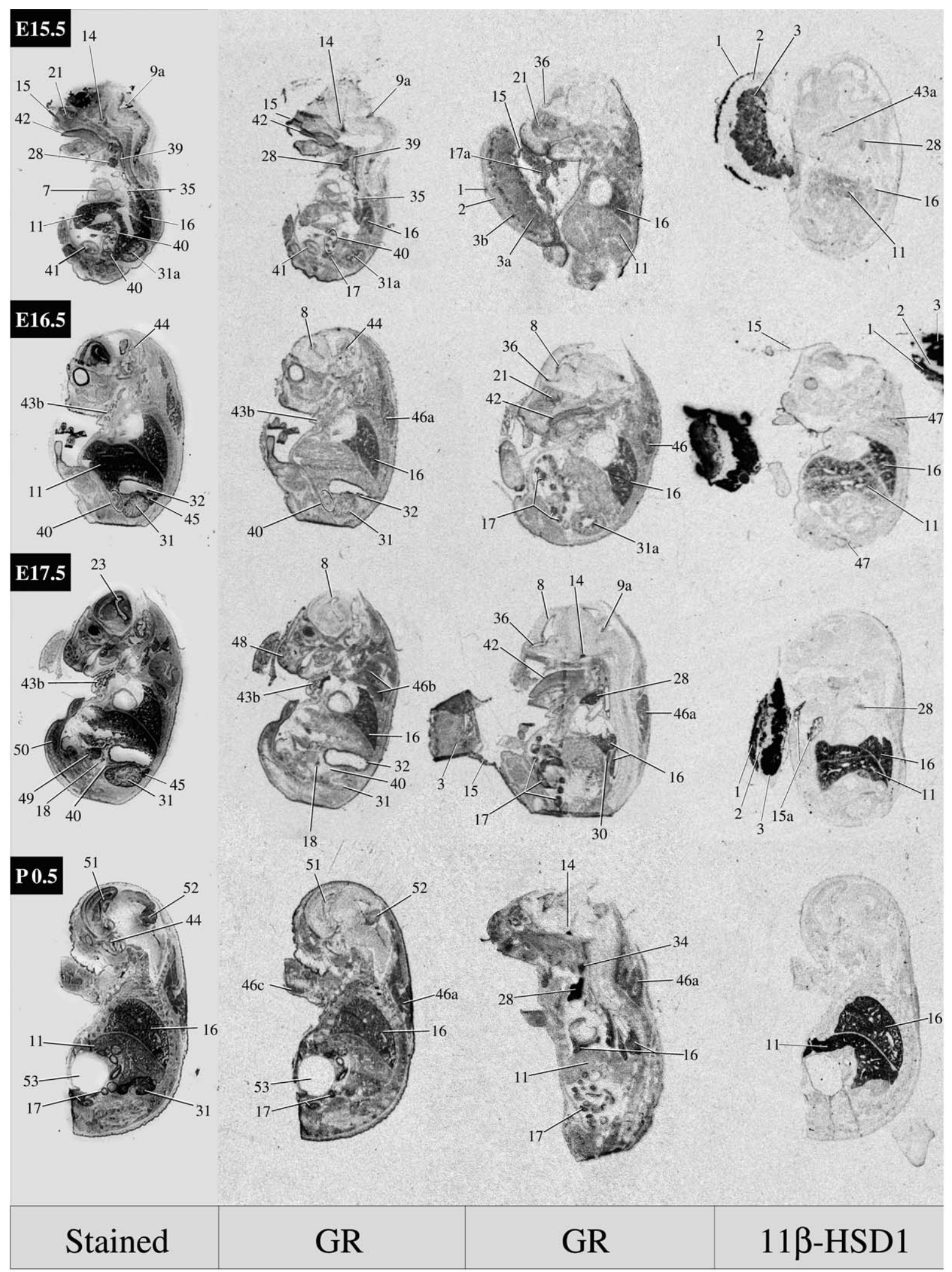

Figure 2(b). 


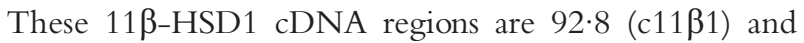
$92 \cdot 0 \%(\mathrm{c} 11 \beta 1 \mathrm{~A})$ identical to the rat $11 \beta-\mathrm{HSD} 1$ sequence (J05107 bp 1-419 and 1-175 respectively). Generally $11 \beta-H S D 1$ mRNA has been shown to overwhelmingly derive from one transcript, 11 $\beta-H S D 1-A$, which has full potential to generate active $11 \beta-H S D 1$ enzyme. However, in one context (adult kidney), a variant truncated transcript, 11 $\beta-H S D 1-B$ (unable to make active $11 \beta$ HSD1 enzyme (Obeid et al. 1993)), has been shown in rat kidney to be as abundant as the normally dominant 11ß-HSD1-A transcript (Krozowski et al. 1990, Moisan et al. 1992). Probes from c11 $\beta 1$ detect $11 \beta-H S D 1$ mRNA very well and have been used throughout these studies. Probes from c11 $\beta 1 \mathrm{~A}$ (which also detect $11 \beta 1 \mathrm{~A}$ but not any truncated $1 \mathrm{~B}$ transcripts) were used to compare with c11 $\beta 1$ data to identify any sites (especially in the kidney) of major discrepancy between total 11 $\beta-H S D 1$ and $11 \beta-$ HSD1-A expression (which would suggest important expression of a non-1A $11 \beta-H S D 1$ transcript).

\section{In situ hybridisation}

Sagittal $10 \mu \mathrm{m}$ cryostat sections were thaw-mounted onto 3-amino propyl triethoxysilane-coated slides and stored at $-80^{\circ} \mathrm{C}$. Tissue sections were fixed, prehybridised, hybridised and washed as described previously (Macdonald et al. 2000; hybridisation was with $\approx 3 \times 10^{6}$ c.p.m./ section ${ }^{35} \mathrm{~S}-\mathrm{UTP}-\mathrm{labelled} \mathrm{RNA}$ probe at $50{ }^{\circ} \mathrm{C}$ for $12-$ $14 \mathrm{~h}$ ). Following washing/RNase A treatment slides were dehydrated, dried and placed against $\beta$-Max Hyperfilm film (Amersham) for 3-14 days to obtain a satisfactory range of film exposures for full analysis. Slides were then dipped in photographic emulsion and exposed in a lighttight box, before being developed as described previously (Macdonald et al. 2000) with the optimal emulsion exposure time (for 18-28 days) determined by the correspond- ing film autoradiographs. The developed slides allowed examination of the gene expression pattern (of silver grains) at microscopic level. In situ hybridisation studies were repeated allowing confirmation of findings in different experiments and different embryos. For GR, almost all findings were confirmed across at least three in situ hybridisation experiments at every gestational stage. Counterstaining, with cresyl violet, was used as necessary to assist in localising expression. Graphical GR and $11 \beta$ HSD1 gene expression profiles for a number of tissues have been derived using greyscale calibration of in situ hybridisation autoradiographs (to those of co-exposed radioactive microscales designed for this purpose (RPA504 and RPA511, Amersham)). Intensive image analysis of the 11ß-HSD1 and especially GR in situ hybridisation autoradiographs was carried out. Autoradiographic films were scanned on a high-resolution flat-bed scanner, into tagged image format files; these were then loaded into AIDA 2.0 image analysis software (Raytek Scientific Ltd, Sheffield, UK). Two-dimensional densitometric analysis was performed allowing precise expression level data, in terms of greyscale values, to be gathered on over 2500 separately demarcated areas of known tissue type and gestation. As previously described (Macdonald et al. 2000, Hou et al. 2002) this calibration of in situ autoradiograph film greyscale values to a common expression level scale (divided into arbitrary units based on the radioactive microscales) permits amalgamation and relative quantitation of the gene expression level for each gene across different films, tissues and gestations. The amalgamated data were used to generate tissue-specific expression profiles having between 3 and 30 measurements at each point; individual curves were generated by least-squares line fitting of the pooled data and the resultant profiles shown in Fig. $3 a$ for GR and Fig. $3 b$ for $11 \beta$-HSD1 assist by illustrating, in multiple

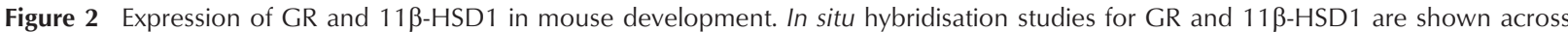
the developmental period studied (E9-5-P0·5). (a) early expression pattern of GR prior to induction of significant fetal $11 \beta$-HSD1 expression, E9.5-E14.5; (b) Gene expression of GR and 11ß-HSD1 from E15.5 to postnatally. For each panel the gestation is shown on the left of the row and the study type (histological staining (cresyl violet) or GR or 11ß-HSD1 in situ hybridisation) is given at the bottom of the column. Autoradiographic exposure is for 6 days with the exception of E9.5 when fetal GR gene expression is faint and thus an exposure at 14 days ((a), E9·5, right panel) is also shown. Apart from extra-embryonic structures, labelling in the in situ hybridisation panels is restricted to areas showing higher expression levels. The areas indicated are: 1, uterine wall; 2, decidua; 3 , fetal zone of placenta (3a, labyrinthine zone; 3b, spongiotrophoblast zone); 4 , chorionic plate; 5 , amniotic fluid cavity; 6 , primitive gut (6a, gut in region of foregut-midgut junction; 6b, ventral foregut in region of floor of pharynx); 7 , heart; 8 , periventricular neuroepithelium; 9 , cerebral fourth ventricle (and associated choroid plexus, 9a); 10, somite elements; 11, developing liver; 12, first branchial arch (12a, migrating thyroid primordium); 13, epithelium of developing pharyngeal wall; 14, developing pituitary; 15, extra-embryonic membranes (15a, membranes surrounding umbilical cord); 16, developing lung; 17, bowel loops (17a, midgut loops in physiological hernia; 17b, distal hindgut-rectal/anal canal); 18, mesonephric duct/ductus deferens; 19, spinal cord; 20, bowel mesentery to physiological hernia; 21, nasal epithelia; 22, septum; 23, lateral ventricle (and associated choroid plexus, 23a); 24, anterior thalamus; 25, ventral isthmus region; 26, ventral pons/medulla; 27, developing larynx (27a, initial oesophagus; $27 \mathrm{~b}$, arytenoid swelling separating trachea and initial oesophagus; 27c, tracheal diverticulum); 28, thymus; 29, heart; ventricular muscle; 30, oesophagus; 31, developing kidney (mesonephric) (31a, ureteric ducts); 32, stomach; 33, striatum (basal ganglia) (33a, striatonuclear neuroepithelium; 33b, striatum-pallidum junction); 34, thyroid; 35 , bronchus; 36, olfactory bulb neuroepithelium; 37, mesencephalic vesicle; 39, upper respiratory tract; 40, developing pancreas (within ellipse); 41, urinary bladder; 42, epithelium of tongue; 43, salivary glands (43a, sublingual; 43b, submandibular); 44, developing cochlea/inner ear; 45, adrenal gland; 46, muscle groups (and specifically 46a, prevertebral; 46b, limb-girdle; 46c, forelimb muscles); 47, muscle group tendons/ fascial planes; 48, whisker follicles (vibrissae); 49, testis; 50, tail; 51, hippocampus; 52, cerebellum; 53, artifactual space. Actual embryo crown-rump length: E9·5, 3 mm; E18·5, 22 mm.
} 
tissues, the trends of expression level of these genes during gestation.

\section{Results}

Control sense $11 \beta-H S D 1$ and GR in situ hybridisations showed non-specific background radioactivity only, which was low for GR but more significant, though uniform across tissues, for $11 \beta-H S D 1$ and $11 \beta-H S D 1 A$. In contrast, antisense studies were characterised by high levels of highly specific hybridisation with low background levels. Multiple sections at each gestation were analysed and for GR in particular this involved confirming findings at each stage across at least three separate in situ hybridisation experiments. Figures 1 and 2 show results of in situ hybridisation from representative sections of the stages studied. Because of the more complex ontogeny of GR, Fig. 2 shows two sections at each gestational stage from E11.5 onward. Sections are in the sagittal plane and after exposure for 6 days with the exception of E9.5 when fetal GR gene expression is faint and thus an exposure at 14 days is also shown. To show a greater range of structures, the sagittal sections used vary in their distance from the midline, though at all stages at least one section passes through the kidney or its embryonic antecedents. Expression profiles by gestational age are illustrated in Fig. $3 a$ (for GR) and $b$ (for 11 $\beta$-HSD1) for multiple tissues. The profiles are calibrated against common index units derived from co-exposed radioactive microscales. Expression is also shown in further detail at sites of particular interest in Figs 4 (for GR) and 5 (for 11 $\beta-H S D 1)$.

\section{Ontogeny of GR}

At E9·5, the uterine wall and the developing fetal zone of the placenta express GR mRNA much more strongly than the maternal (decidual) zone of the placenta or the very low expression prevailing in the fetus at this stage of gestation, with the notable exception of the ventral foregut (see below). At later gestational ages, placental expression shows very little change with the fetal $>$ decidual zone pattern persisting, while expression clearly rises in the fetus. This rise in overall fetal GR gene expression (average expression across all fetal tissues) from the low levels of E9.5 is well developed by E11.5 and gradually continues until after birth. However it is abundantly clear (see Fig. 2) that this rising fetal GR gene expression is not uniform; there are striking differences across fetal tissues both in the extent and the pattern of increase. Indeed, in a few tissues there is a fall in GR mRNA later in gestation. The ontogeny of GR gene expression is considered in detail below for two periods: from E9. 5 to E14 and then from E14.5 to postnatally.

E9-5-E14 As noted above, early- to mid-gestation GR gene expression is generally very low but by E9-E9. 5 it is clearly higher in the ventral foregut (floor of pharynx). From E9.5 to E14.5 (especially by E12.5) this higher expression in the pharynx increases further and spreads in all directions around the developing gut, including to the pouches and diverticulae, then branching from it to involve: (i) all foregut, midgut and hindgut by E11.5; (ii) the dorsal foregut involving Rathke's pouch by E11.5E12 and shortly afterwards the primitive anterior pituitary (from E12, Fig. 4b) and spreading also more proximally into early nasal cavities (from E12.5-E13.5 onwards); (iii) the branchial arches/pouches notably the second (hyoid) arch (contributing to midline tongue) and the third and fourth branchial pouches (all, E12-E12.5) with high expression following in the thymus (a third pouch derivative) clearly seen separately at E12.5-E13 (though the thymus is still small); and (iv) important ventral foregut diverticulae involving the developing thyroid, trachea and early bronchial tree and most dramatically the hepatic diverticulum which initiates liver development on infiltrating into septum transversum (E10.5-E12). The pancreatic diverticulae are present from $\sim$ E9.5-E10, but GR gene expression remains very low/absent before E14 (see below and Fig. 4a). Thus, the most dramatic increase of the E9.5-E12.5 period is that in hepatic primordia especially E10.5-E12 (see Fig.2, E11.5), a phase during which the liver is invaded by haematopoietic stem cells and first takes over a major haematopoietic role. Across E12-E13.5 hepatic expression declines somewhat. In contrast, expression in the upper GI and respiratory tract rises further while increased GR gene expression is first clearly seen in: (i) mesonephric tubules and duct (E11.5-E12.5) and from E12.5/E13.5 onwards in the forming primitive definitive kidney (metanephros) and the ureteric duct and its branches; (ii) precartilage condensations at sites of imminent cartilage and later bone development, most notably in the developing vertebral column (E12-E13, declining by E13.5 with cartilage formation); and (iii) discrete regions of the CNS ( $\sim$ E12 onwards) particularly hypothalamus, the ventral aspect of the pons-medullarostral spinal cord, the ventral isthmus/midbrain, strongly in choroid of lateral and fourth ventricles and at somewhat lower levels in periventricular neuroepithelium more generally.

E14.5-postnatal From E14.5 until term the majority of fetal tissues show a gradual, modest further incrase in GR gene expression levels with rises to substantial expression levels in several tissues. As the expression in the fetal placental zone remains at an approximately constant level across this period, this level serves as a useful reference threshold with the following tissues showing higher GR gene expression levels for the durations indicated: (i) pharynx, upper respiratory tract and bronchial tree (from $\sim \mathrm{E} 12.5$ onwards) and lung (E14.5-E15 onwards until $\mathrm{P} 0 \cdot 5$ (with an additional sharp rise at E18.5 falling back by $\mathrm{P}(\cdot 5)$ ); (ii) intestine continuing from $\mathrm{E} 12 \cdot 5$ 
a
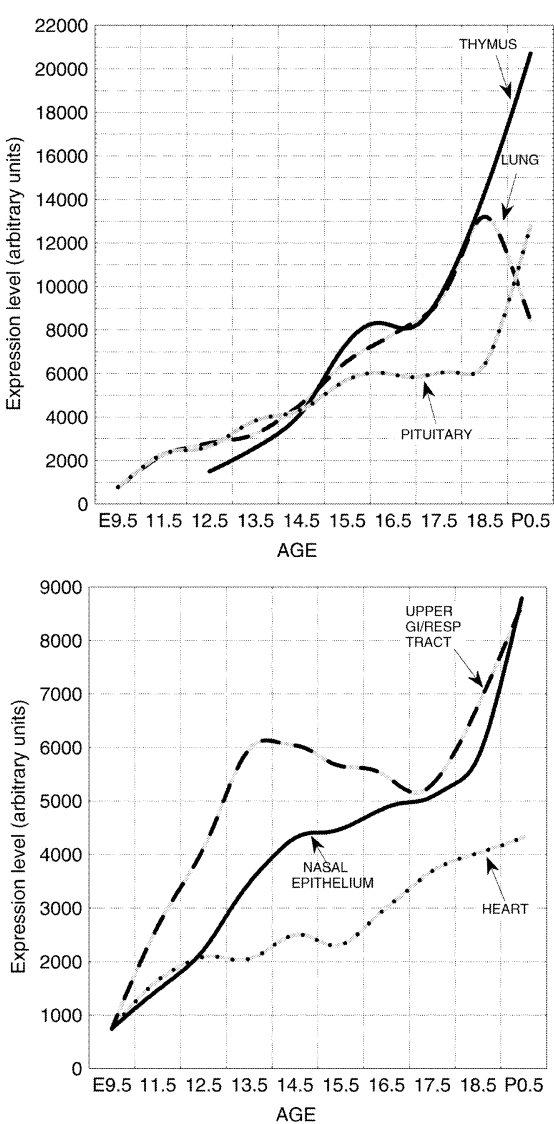

b

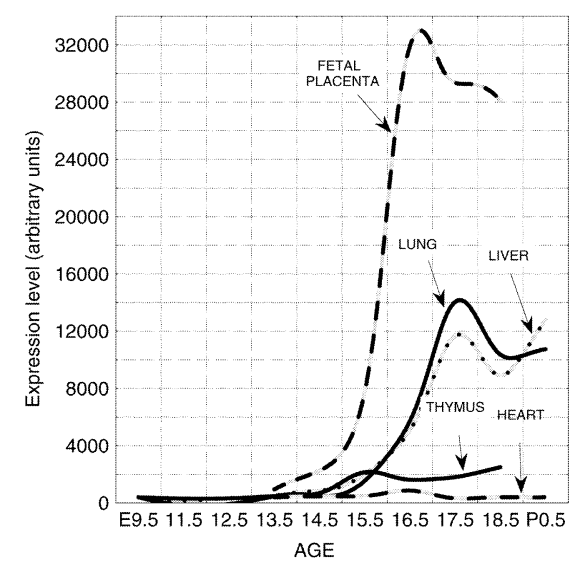

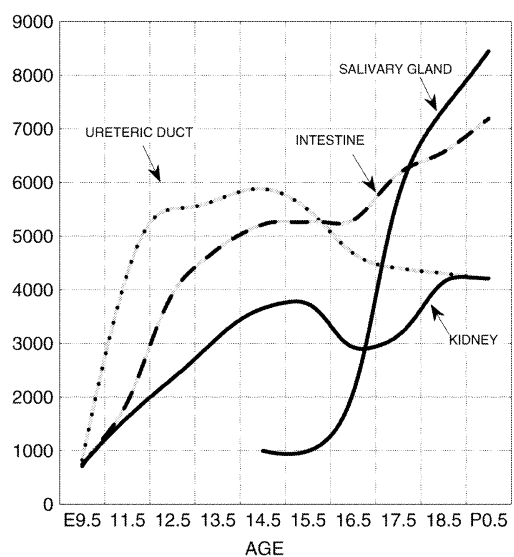

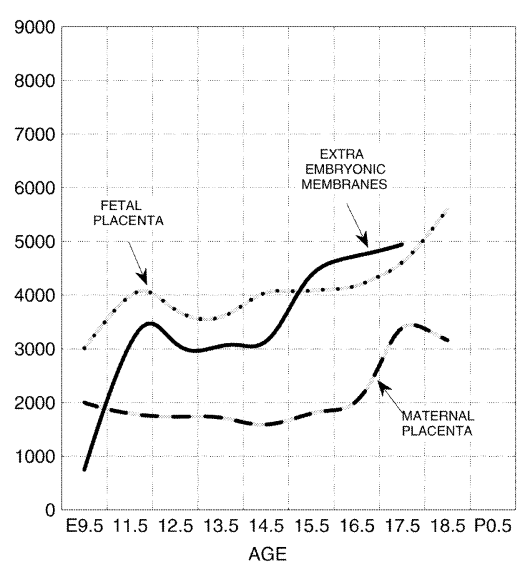

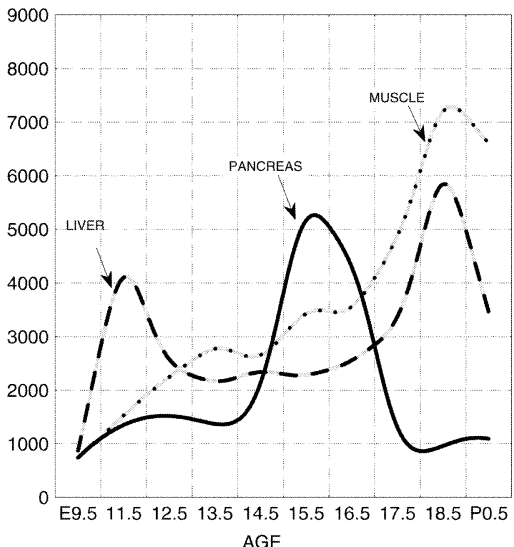

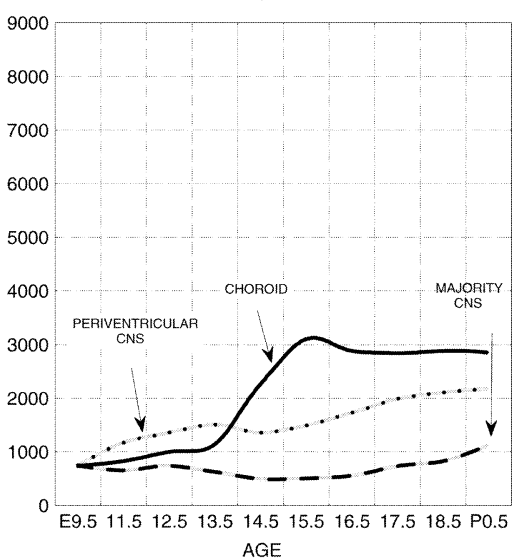

Figure 3 (a) Developmental GR gene expression profiles for mouse tissues. These were generated from image analysis densitometry data, calibrated to co-exposed radioactive microscales, allowing expression from all in situ hybridisation studies (not just those used for illustration in Figure 2) to be pooled and profiles generated by least-squares curve fitting. Profiles from 19 organs/tissues are shown. Note that the scaling of the top-left graph differs from all others in going to a much higher expression level and this graph profiles the most highly expressing tissues. Ureteric ducts are shown separately while expression over the kidney, except for ureteric ducts, is designated kidney. Upper Gl/Resp Tract refers to oropharynx, larynx/trachea and upper oesophagus. Periventricular CNS is shown separately from the choroid plexus and the expression level average over the rest of the CNS (designated Majority CNS). The purpose here is to identify trends, not to make quantitative comparisons, as the analysis is semi-quantitative at most. Nonetheless two qualifications are useful: (i) it appears that as the expression level drops below $\sim 750$ arbitrary units it is not significantly different from background; (ii) changes of $<30-50 \%$ cannot be regarded as robust but become increasingly so above this level and when trends continue across multiple gestational ages. (b) Developmental 11 $\beta$-HSD1 gene expression profiles for mouse tissues (see panel (a) legend). Other major organs/tissues not shown had very little or no convincing evidence of expression across the organ as a whole. 

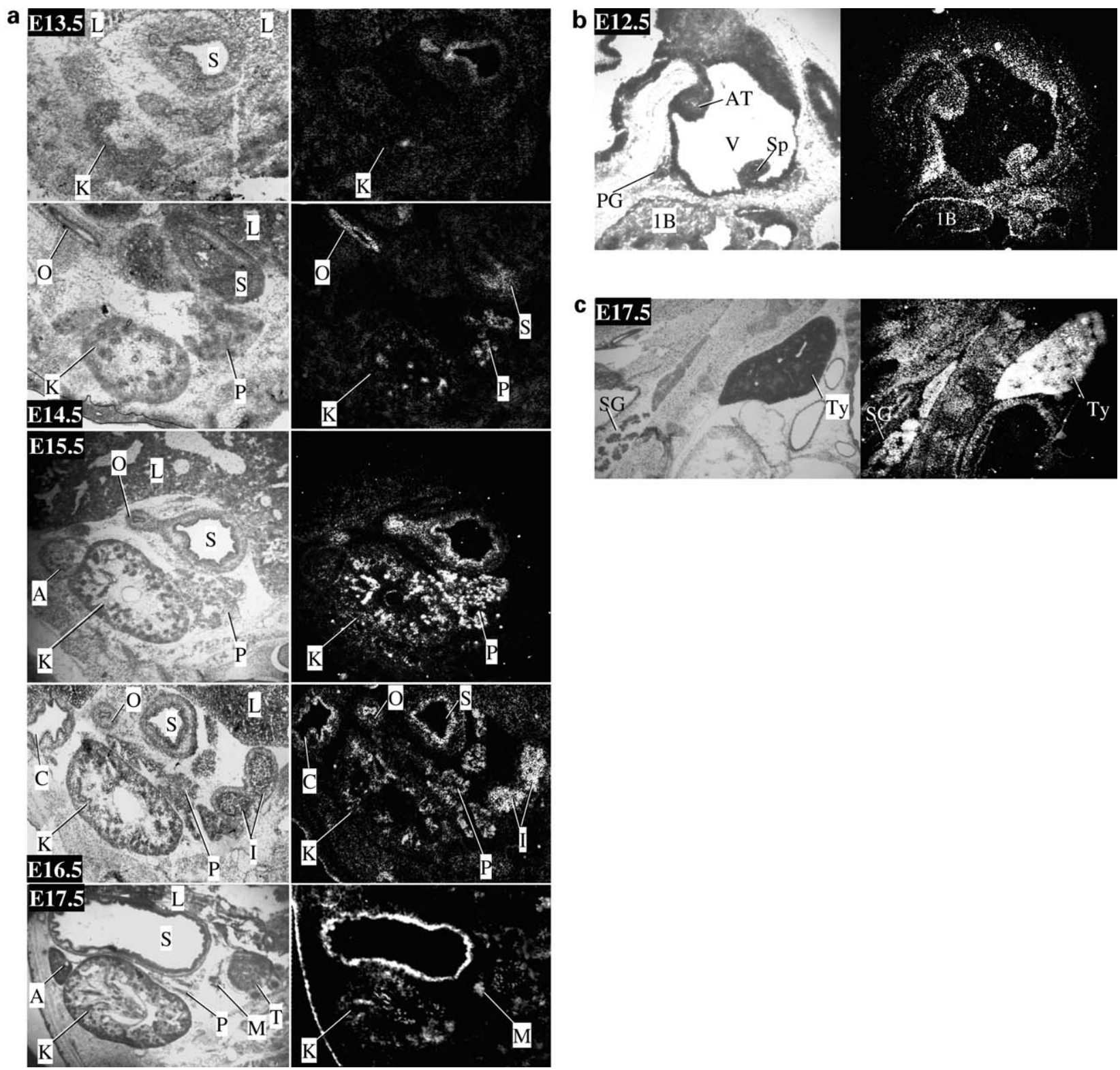

Figure 4 Detail of GR gene expression at sites of particular interest. (a) Changes in GR gene expression in stomach, pancreas and kidney; (b) early GR gene expression in the region of the developing pituitary and hard palate; (c) abundant GR gene expression in the developing thymus and salivary gland. Right-hand panels show dark-field in situ hybridisation findings and left-hand panels show corresponding appearance with cresyl violet histological staining and indicate the gestational stage. $1 \mathrm{~B}$, first branchial arch; A, adrenal gland; AT, anterior thalamus; C, colon; I, intestine; K, kidney; L, liver; M, mesonephric duct/ductus deferens; O, oesophagus; P, pancreas, PG, pituitary gland; S, stomach; SG, salivary (submandibular) gland; Sp, septum; T, testis; Ty, thymus.

through to postnatal; (iii) liver briefly at E10.5-E12 and again briefly at E18.5, dropping between these days and postnatally; (iv) pancreas at E14.5/15 until E16.5 with lower expression before and afterwards; (v) muscle from E16.5-E17 onwards especially in erector spinae and posterior cervical musculature; (vi) ureteric duct/collecting ducts from E13.5 to E16.5, thereafter declining to lower levels then prevailing more widely in the kidney; (vii) thymus from E13.5, rising sharply higher again from E18 to P0.5; (viii) pituitary from E14.5/E15 onwards, also rising sharply higher again from $\mathrm{E} 18 \cdot 5$ to $\mathrm{P} 0 \cdot 5$; (ix) salivary gland from E17 onwards; and (x) nasal epithelium from E14.5 onwards and rising further sharply postnatally. In all these cases expression is well above the average across all fetal tissues and the increases which develop in early upper respiratory/GI tract, progressively 


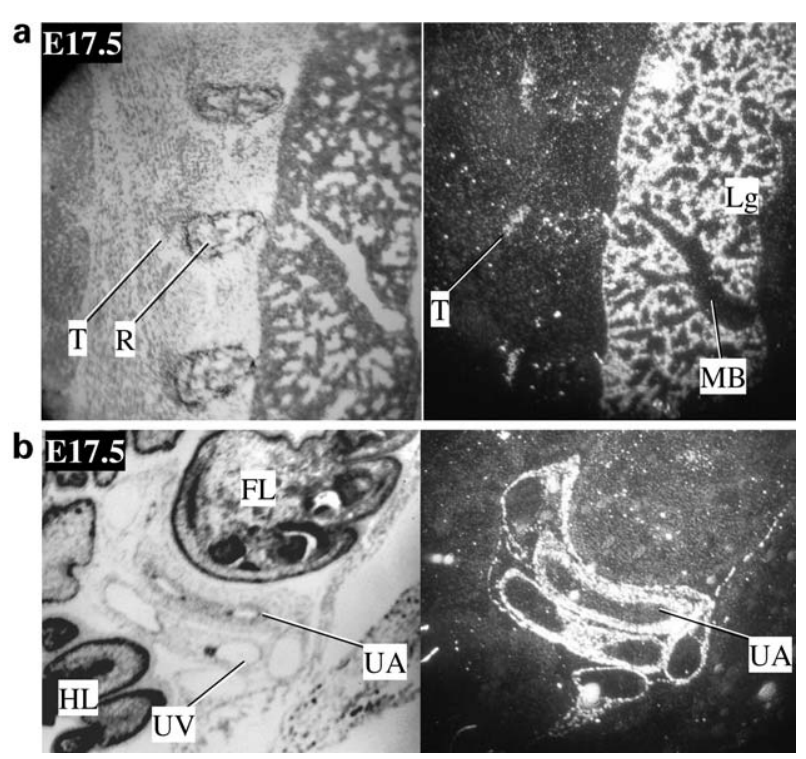

Figure 5 Detail of $11 \beta$-HSD1 gene expression at sites of particular interest. (a) GR gene expression in the region of the umbilical cord; (b) Early GR gene expression in the paravertebral/posterior rib-cage region. Right-hand panels show dark-field in situ hybridisation findings and left-hand panels show corresponding appearance with cresyl violet histological staining and indicate the gestational stage. $\mathrm{FL}$, forelimb digits; $\mathrm{HL}$, hindlimb; $\mathrm{Lg}$, lung; $\mathrm{MB}$, major bronchus; R, developing rib; $\mathrm{T}$, tendon; UA, umbilical artery; UV, umbilical vein.

in lung, pituitary and thymus and transient in developing pancreas (see also Fig. $4 a$ ) are especially striking.

In contrast, GR mRNA expression remains low in: (i) the decidual zone of the placenta; (ii) spinal cord and the majority of CNS regions which are not periventricular; (iii) gonads; (iv) adrenal; (v) the pancreas from E17 onwards; (vi) regions with cartilage formation especially in the developing vertebral column from E14 onwards. Regions with intermediate GR gene expression levels (being higher than the six sites just listed but lower than the fetal zone of the placenta) include heart, liver (between E13 and E17) and the majority of periventricular neuroepithelial regions of the CNS, basal ganglia, choroid plexus and later at lower levels in olfactory neuroepithelium, cerebellum and hippocampus.

Of special interest is GR expression in the pituitary which participates in functional hypothalamo-pituitaryadrenal (HPA)-axis feedback from at least as early as E16-E16.5 (Reichardt \& Schutz 1996). As can be seen in Fig. $4 b$, abundant GR mRNA is present by E12 and it is between these gestational days that fetal adrenal glucocorticoid production commences. GR in structures located inferiorly to the developing pituitary fossa also play key roles in palatal fusion. The developing thymus is known to be susceptible to glucocorticoid-driven apoptosis, playing an obvious potentially important role in the maturation of immune responses. As seen in Fig. 2, GR expression develops strongly between E14.5 and E17.5. By E17.5 the thymus is also expressing moderate levels of $11 \beta-\mathrm{HSD} 1$ mRNA as well as abundant GR expression (Fig $4 c$ ).

\section{Ontogeny of 11/-HSD1}

Fetal 11 $\beta-H S D 1$ mRNA expression is first convincing at $\sim$ E13, initially in the fetal placenta. Fetal $11 \beta-H S D 1$ expression is seen as follows: (i) from $\sim \mathrm{E} 15$ in lung and liver then rising strongly until after birth. It is seen at lower levels in: (ii) the thymus, from E15; (iii) a few brain regions (more limited than rat (Diaz et al. 1998)) including hippocampus (especially after E16); (iv) structures in the umbilical cord which are strongly expressing by E17.5 (see Fig. $5 a$ ) at a time when $11 \beta-H S D 1$ has increased to high levels in the fetal zone of the placenta and in the maternal uterine wall proper (but not the decidual (maternal zone) of the placenta); and finally (v) $11 \beta-H S D 1$ is expressed strongly in discrete sites in developing muscle beds (limb girdle, paraspinal, rib-cage and neck) from E16.5 to P0.5, this appears to correspond to points of attachment of tendons. Figure 2 indicates some examples in limb girdle and neck muscle beds and this is shown for the rib-cage in Fig. $5 b$. Comparison of $11 \beta-H S D 1$ expression using c11 $\beta 1$ and $c 11 \beta 1 \mathrm{~A}$ probes revealed no major discrepancies in detected 11 $\beta-$ HSD1 distribution indicating no evidence for significant levels of truncated (11 $\beta-H S D 1-B)$ transcripts throughout ontogeny, including in the kidney. Hence the major 11 $\beta-H S D 1$ transcript throughout ontogeny is $11 \beta-H S D 1-A$, a transcript with full potential to make $11 \beta-H S D 1$ enzyme.

\section{Discussion}

We have examined the bodywide expression of GR and $11 \beta$-HSD1 (both determinants of potential tissue glucocorticoid sensitivity) in mouse from mid-gestation (E9.5) to just postnatal. For most organ systems this period parallels the major proportion of human gestation from $\leq 6$ weeks gestation to just after birth (O'Rahilly \& Muller 1996, Bard \& Kaufman 1999). Some previous work has examined expression of one or both genes in a considerably more limited way either focusing in detail on a single organ system or a few developmental time points (Condon et al. 1998, Diaz et al. 1998, Waddell et al. 1998). This is the first study to determine the ontogeny of both genes throughout the body in detail during such a major phase of development up until just after birth. Moreover, this current work is directly complementary to our previous similar studies of MR and $11 \beta-\mathrm{HSD} 2$ gene expression (Brown et al. 1996c), two further key determinants of the potential for tissue sensitivity to corticosteroid hormones. Of course, to establish definitively whether high tissue sensitivity to these hormones is indeed manifest requires additional studies beyond the scope of the current work, 
especially demonstrating levels of hormone-specific physiological responsiveness. However, the current studies provide a useful starting point.

The prenatal expression pattern of $11 \beta-H S D 1$ (an enzyme producing active glucocorticoid) is very simple compared with GR. 11 $\beta-$ HSD1 expression is first seen at $\mathrm{E} 13$ in the fetal (labyrinthine) zone of the placenta, increasing in level towards birth; however, expression of 11ß-HSD2 (Brown et al. 1996c), which inactivates glucocorticoids, drops from high to low or undetectable levels in many fetal tissues between E12.5 and E13.5 and in the fetal zone of the placenta between E14.5 and E16. Thus this zone of the placenta, which is the interface between maternal and fetal circulations, transforms over E13-E16 from a tissue potently inactivating glucocorticoids to one in which the gene expression supporting this (11 $\beta-H S D 2)$ switches off while expression supporting the production of glucocorticoid (11ß-HSD1) switches on. The adjacent maternal zone of the placenta, which has other roles, shows no convincing overall expression of either $11 \beta-\mathrm{HSD} 1$ or 11 $\beta-H S D 2$ (Brown et al. 1996c). A recent study on mouse placenta reports similar findings and discusses them in some detail (Thompson et al. 2002).

GR and 11 $\beta$-HSD1 have proved to be genes where mRNA expression reflects well the expression of the functional protein provided that the non-functional transcripts (GR $\beta$ (Oakley et al. 1996) and 11ß-HSD1-B (Krozowski et al. 1990, Moisan et al. 1992, Obeid et al. 1993)) are not proportionately abundant. The in situ hybridisation probes used in the work above selectively detect the functional GR $\alpha$ transcript, moreover GR $\beta$ is not expressed in the mouse (Otto et al. 1997). Similarly, we have shown no major discrepancies between the $11 \beta-H S D 1$ gene expression and that of the active $11 \beta-$ HSD1-A transcript. $11 \beta-$ HSD1 gene expression is minimal or absent up to $\mathrm{P} 0 \cdot 5$ in the kidney (the organ where $11 \beta-$ HSD1-B has been found in other species).

The results above predict high glucocorticoid sensitivity in some tissues, especially lung (abundant GR and $11 \beta-H S D 1$ ) and thymus (abundant GR and moderate $11 \beta-H S D 1)$. Evidence of such sensitivity is indeed present (including physiological responses, e.g. lung maturation, thymocyte apoptosis) from previous work in several species including human, rodents and sheep (Dodic \& Wintour 1994, Ballard 2000, Forhead et al. 2000). To show the strength of this evidence it is valuable to describe a good example of this in some detail. Thus, for example, fetal lung maturation is strongly accelerated merely by maternal glucocorticoid administration in later gestation in humans and in animals that have been studied (Ballard 2000). Conversly the 'GR knockout' mouse dies in the neonatal period because of lung immaturity; this situation cannot be avoided by the use of synthetic glucocorticoids as GR is lacking (Cole et al. 1995). Furthermore, our gene expression work predicts a varying glucocorticoid sensitivity of developing lung which again appears borne out in its responsiveness in several species (Ballard 2000). Specifically, our previous work indicated that before E13 in mouse there is high $11 \beta-\mathrm{HSD} 2$ gene expression in many fetal tissues potentially representing sites of eradication of, and insensitivity to, glucocorticoids (Brown et al. 1996c). By E13.5, this $11 \beta-H S D 2$ expression sharply downregulates in many tissues including lung, where $11 \beta-$ HSD2 mRNA is minimal/absent by E15. The present work shows that in E15-E15.5 lung GR gene expression rises and $11 \beta-H S D 1$ is first expressed and then also rises sharply. These findings clearly suggest relative insensitivity to physiological glucocorticoids in the lung up to E13, diminishing over E13.5 with strongly increasing glucocorticoid sensitivity from E15. This is supported by animal and human studies. Abundant 11 $\beta$-HSD2-like glucocorticoid inactivating activity is reported as late as embryonic week (EW) 21 in the human lung (Murphy 1978) with $11 \beta-H S D 2$ present before this time (Condon et al. 1998) but absent by 22-23 weeks (Brown et al. 1996b). Benefits accelerating lung maturation with glucocorticoid treatment are clear from EW26 and probably diminish somewhat going back to EW22-23 (the lower limit of viability for premature babies) (Ward 1994). Glucocorticoid treatment in mice with comparable lung development (E14E15.5) accelerates lung maturation; however, stronger treatment on both E14.5 and E15.5 increases lung maturity but also reduces mature lung and lung/body weight ratio; this effect is permanent (Stewart et al. 1998), reflecting concerns about glucocorticoid overtreatment prenatally in humans (Lacaze-Masmonteil \& Audibert 2000, Seckl et al. 2000). There is also ample evidence, from work in the fetal sheep, of glucocorticoid sensitivity in the lung and acceleration of lung maturation by such treatment (Ballard 2000, Forhead et al. 2000).

Some other sites with expression indicating increasing glucocorticoid sensitivity, e.g. bowel and periventricular CNS, are also now seen as sites where antenatal glucocorticoid can accelerate maturation in the human premature infant and reduce harmful sequelae such as periventricular leukomalacia (Canterino et al. 2001) (a major aetiological risk factor for cerebral palsy) and impaired bowel motility (especially of the duodenum (Morriss et al. 1986)). These parallels between our studies and the functional glucocorticoid sensitivity of human and rodent organs (e.g. lung), give some support to the more general usefulness of the detailed conclusions which can be drawn from the results above. Of course conclusions that such tissues may be especially responsive to glucocorticoids would require further experimental work to assess. The current studies suggest likely sites and critical periods where heightened glucocorticoid sensitivity may have particularly longlasting effects on ureteric ducts, the early skeleton, some CNS regions and especially GI and respiratory tracts, pancreas, pituitary-hypothalamus and thymus. The detailed role of glucocorticoids in development at the majority of these sites has not been directly ascertained but 
often previous literature points to glucocorticoids having a role, especially in the transition to a more differentiated less proliferative state.

One of the more intriguing of such sites is the developing pancreas which by $\sim$ E9.5-E10 has arisen from the foregut as two progenitor buds which proliferate strongly and fuse at E13-E14. In the pancreas GR mRNA is at a very low level up to E13.5-E14, increasing steeply to much higher levels between E14.5 and 16.5, a period coinciding with two important changes in pancreatic development. First, from E14.5, acini and ducts are readily discernable histologically, representing the transition to a less proliferative more differentiated phase. Secondly, until E14.5-E15.5 cells destined to form islets are largely scattered and intraductal but during E15.5-E16.5 clusters migrate out of duct epithelium, forming islets from E16.5 onwards. The abundant GR mRNA expression observed at E14.5-E16.5 is too widespread to be solely in islet progenitor cells and must represent expression in the majority of ductal cells; however, it is impossible to discern in the current work if this includes or excludes the rare islet progenitor cells.

Glucocorticoid action on the developing pancreas has not been extensively studied; however, previous work on the immature pancreas (Lu et al. 1987, Komatsu et al. 1998), poorly differentiated pancreatic cancers (Norman et al. 1994) and largely exocrine pancreatic cell lines (Rosewicz et al. 1991, Slater et al. 1993) shows clear evidence of GR expression and that glucocorticoids reduce proliferation rate (Norman et al. 1994) and alter gene expression via GR (Rosewicz et al. 1991, Slater et al. 1993). Indeed dexamethasone is a useful component of chemotherapy for poorly differientated pancreatic cancers (Norman et al. 1994). Literature on the more mature pancreas indicates GR expression in islets (Matthes et al. 1994) (co-localising exclusively to $\beta$-cells (Fischer et al. 1990)) while GR in exocrine pancreas is much reduced (Matthes et al. 1994). Finally, one study on rats suggested that maternal adrenal steroids were required to maintain a normal rate of pancreatic $\beta$-cell growth in the developing fetus, with defects seen at or before E15 with maternal adrenalectomy (Komatsu et al. 1998). Another study showed reduced pancreatic weight (and some other organ weights reduced too) after late-gestational glucocorticoid treatment in monkeys (Johnson et al. 1981). The results above and these previous reports strongly suggest that if glucocorticoid exposure of the developing pancreas is inadequate or excessive then the function of the mature organ will be altered. This is especially intriguing in view of the fact that late-prenatal dexamethasone causes permanently impaired glucose tolerance in the offspring as adults (Seckl et al. 2000).

The results of this work should thus help to identify sites that are particularly vulnerable to the effects of glucocorticoid excess, either exogenous (maternally administered) or arising endogenously from substantial maternal (or fetal) stress. Clearly further careful direct study is required to define properly what effect glucocorticoid and its excess have on these potentially vulnerable tissues, such as the developing pancreas. Nonetheless, these findings give some assistance in the investigation of how glucocorticoids influence fetal programming and may give insights into the association, shown in humans, between low birth weight and subsequent diabetes, hyperlipidaemia and hypertension (Barker 1994, Seckl et al. 2000).

\section{Acknowledgements}

We thank Susan Coan and Lynne Ramage for expert technical assistance.

\section{Funding}

This work was supported by grants from the Scottish Hospitals Endowment Research Trust (grant RG77/00), Urquhart Charitable Trust, Wellcome Trust and Medical Research Council.

\section{References}

Ballard PL 2000 Scientific rationale for the use of antenatal glucocorticoids to promote fetal development. Pediatrics in Review $\mathbf{1}$ E83-E90.

Bard JBL \& Kaufman MH 1999 The Anatomical Basis of Mouse Development. Oxford: Academic Press.

Barker DJP 1994 Mothers, Babies and Diseases in Later Life. London: BMJ Publishing Group.

Brown RW, Chapman KE, Kotelevtsev Y, Yau J, Lindsay RM, Brett L, Leckie C, Murad P, Lyons V, Mullins JJ, Edwards CRW \& Seckl JR 1996a Cloning and production of antisera to human placental 11 $\beta$-hydroxysteroid dehydrogenase type 2. Biochemical Journal 313 997-1006.

Brown RW, Chapman KE, Kotelevtsev Y, Yau JLW, Lindsay RS, Brett L, Leckie C, Murad P, Lyons V, Mullins JJ, Edwards CRW \& Seckl JR 19966 Cloning and production of antisera to human placental $11 \beta$-hydroxysteroid dehydrogenase type 2. Biochemical Journal 313 1007-1017.

Brown RW, Diaz R, Robson AC, Kotelevtsev YV, Mullins JJ, Kaufman MH \& Seckl JR 1996c The ontogeny of $11 \beta$-hydroxysteroid dehydrogenase type 2 and mineralocorticoid receptor gene expression reveal intricate control of glucocorticoid action in development. Endocrinology 137 794-797.

Canterino JC, Verma U, Visintainer PF, Elimian A, Klein SA \& Tejani N 2001 Antenatal steroids and neonatal periventricular leukomalacia. Obstetrics and Gynecology 97 135-139.

Cole TJ, Blendy JA, Monaghan AP, Krieglstein K, Schmid W, Aguzzi A, Fantuzzi G, Hummler E, Unsicker K \& Schutz G 1995 Targeted disruption of the glucocorticoid receptor gene blocks adrenergic chromaffin cell development and severely retards lung maturation. Genes and Development 9 1608-1621.

Condon J, Gosden C, Gardener D, Nickson P, Hewison M, Howie AJ \& Stewart PM 1998 Expression of type 211 beta-

hydroxysteroid dehydrogenase and corticosteroid hormone receptors in early human fetal life. Journal of Clinical Endocrinology and Metabolism 83 4490-4497. 
Danielsen M, Northrop JP \& Ringold GM 1986 The mouse glucocorticoid receptor: mapping of functional domains by cloning, sequencing and expression of wild-type and mutant receptor proteins. EMBO Journal 5 2513-2522.

Diaz R, Brown RW \& Seckl JR 1998 Distinct ontogeny of glucocorticoid and mineralocorticoid receptor and 11 betahydroxysteroid dehydrogenase types I and II mRNAs in the fetal rat brain suggest a complex control of glucocorticoid actions. Journal of Neuroscience 18 2570-2580.

Dodic M \& Wintour EM 1994 Effects of prolonged (48 h) infusion of cortisol on blood pressure, renal function and fetal fluids in the immature ovine foetus. Clinical and Experimental Pharmacology and Physiology 21 971-980.

Edwards CRW, Benediktsson R, Lindsay RS \& Seckl JR 1993 Dysfunction of placental glucocorticoid barrier: link between fetal environment and adult hypertension? Lancet 341 355-357.

Fischer B, Rausch U, Wollny P, Westphal H, Seitz J \& Aumuller G 1990 Immunohistochemical localization of the glucocorticoid receptor in pancreatic beta-cells of the rat. Endocrinology 126 2635-2641.

Forhead AJ, Gillespie CE \& Fowden AL 2000 Role of cortisol in the ontogenic control of pulmonary and renal angiotensin-converting enzyme in fetal sheep near term. Journal of Physiology 526 409-416.

Hou J, Speirs HJ, Seckl JR \& Brown RW 2002 Sgk1 gene expression in kidney and its regulation by aldosterone: spatio-temporal heterogeneity and quantitative analysis. Journal of the American Society of Nephrology 13 1190-1198.

Johnson JWC, Mitzner W, Beck JC, London WT, Sly DL, Lee PA, Khouzami VA \& Cavalieri RL 1981 Long-term effects of betamethasone on fetal development. American Journal of Obstetrics and Gynecology 141 1053-1064.

Komatsu S, Yamamoto M, Arishima K \& Eguchi Y 1998 Maternal adrenocortical hormones maintain the early development of pancreatic B cells in the fetal rat. Journal of Anatomy 193 551-557.

Krozowski Z, Stuchbery S, White P, Monder C \& Funder JW 1990 Characterization of 11 beta-hydroxysteroid dehydrogenase gene expression: identification of multiple unique forms of messenger ribonucleic acid in the rat kidney. Endocrinology 127 3009-3013.

Lacaze-Masmonteil T \& Audibert F 2000 Multiple courses of antenatal glucocorticoid treatment and fetal outcome. Journal of Perinatal Medicine 28 185-193.

Lu RB, Lebenthal E \& Lee PC 1987 Developmental changes of glucocorticoid receptors in the rat pancreas. Journal of Steroid Biochemistry 26 213-218.

Macdonald P, MacKenzie S, Ramage LE, Seckl JR \& Brown RW 2000 Corticosteroid regulation of amiloride-sensitive sodiumchannel subunit mRNA expression in mouse kidney. Journal of Endocrinology 165 25-37.

Matthes H, Kaiser A, Stier U, Riecken EO \& Rosewicz S 1994 Glucocorticoid receptor gene expression in the exocrine and endocrine rat pancreas. Endocrinology 135 476-479.

Moisan MP, Edwards CR \& Seckl JR 1992 Differential promoter usage by the rat 11 beta-hydroxysteroid dehydrogenase gene. Molecular Endocrinology 6 1082-1087.

Morriss FH Jr, Moore M, Weisbrodt NW \& West MS 1986 Ontogenic development of gastrointestinal motility: IV. Duodenal contractions in preterm infants. Pediatrics 78 1106-1113.

Mune T, Rogerson FM, Nikkila H, Agarwal AK \& White PC 1995 Human hypertension caused by mutations in the kidney isozyme of 11 beta-hydroxysteroid dehydrogenase. Nature Genetics 10 394-399.
Murphy BE 1978 Cortisol production and inactivation by the human lung during gestation and infancy. Journal of Clinical Endocrinology and Metabolism 47 243-248.

Norman J, Franz M, Schiro R, Nicosia S, Docs J, Fabri PJ \& Gower WRJ 1994 Functional glucocorticoid receptor modulates pancreatic carcinoma growth through an autocrine loop. Journal of Surgical Research 57 33-38.

O'Rahilly RR \& Muller F 1996 Human Embryology and Teratology. Hoboken, New Jersey: Wiley.

Oakley RH, Sar M \& Cidlowski JA 1996 The human glucocorticoid receptor beta isoform. Expression, biochemical properties, and putative function. Journal of Biological Chemistry 271 9550-9559.

Obeid J, Curnow KM, Aisenberg J \& White PC 1993 Transcripts originating in intron 1 of the HSD11 (11 beta-hydroxysteroid dehydrogenase) gene encode a truncated polypeptide that is enzymatically inactive. Molecular Endocrinology 7 154-160.

Otto C, Reichardt HM \& Schutz G 1997 Absence of glucocorticoid receptor-beta in mice. Journal of Biological Chemistry 272 26665-26668.

Reichardt HM \& Schutz G 1996 Feedback control of glucocorticoid production is established during fetal development. Molecular Medicine 2 735-744.

Rosewicz S, Detjen K, Logsdon CD, Chen LM, Chao J \& Riecken EO 1991 Glandular kallikrein gene expression is selectively down-regulated by glucocorticoids in pancreatic AR42J cells. Endocrinology 128 2216-2222.

Seckl JR \& Brown RW 1994 11-Beta-hydroxysteroid dehydrogenase: on several roads to hypertension. Journal of Hypertension $\mathbf{1 2}$ $105-112$.

Seckl JR, Cleasby M \& Nyirenda MJ 2000 Glucocorticoids, 11 beta-hydroxysteroid dehydrogenase, and fetal programming. Kidney International 57 1412-1417.

Slater EP, Hesse H, Muller JM \& Beato M 1993 Glucocorticoid receptor binding site in the mouse alpha-amylase 2 gene mediates response to the hormone. Molecular Endocrinology 7 907-914.

Stewart JD, Sienko AE, Gonzalez CL, Christensen HD \& Rayburn WF 1998 Placebo-controlled comparison between a single dose and a multidose of betamethasone in accelerating lung maturation of mice offspring. American Journal of Obstetrics and Gynecology 179 1241-1247.

Thompson A, Han VKM \& Yang K 2002 Spatial and temporal patterns of expression of $11 \beta$-hydroxysteroid dehydrogenase types 1 and 2 messenger RNA and glucocorticoid receptor protein in the murine placenta and uterus during late pregnancy. Biology of Reproduction 67 1708-1718.

Waddell BJ, Benediktsson R, Brown RW \& Seckl JR 1998 Tissue-specific messenger ribonucleic acid expression of 11 beta-hydroxysteroid dehydrogenase types 1 and 2 and the glucocorticoid receptor within rat placenta suggests exquisite local control of glucocorticoid action. Endocrinology 139 1517-1523.

Ward RM 1994 Pharmacologic enhancement of fetal lung maturation. Clinics in Perinatology 21 523-542.

Whorwood CB, Franklyn JA, Sheppard MC \& Stewart PM 1992 Tissue localization of 11 beta-hydroxysteroid dehydrogenase and its relationship to the glucocorticoid receptor. Journal of Steroid Biochemistry and Molecular Biology 41 21-28.

Received 26 November 2003

Accepted 9 December 2003 